\title{
Reliability and Validity of NX-16 3D Body Scanner
}

\author{
Confiabilidad y Validez del Escaner Corporal 3D NX-16
}

\author{
Jozef Simenko* \& Ivan Cuk**
}

SIMENKO, J. \& CUK, I. Reliability and validity of NX-16 3D body scanner. Int. J. Morphol., 34(4):1506-1514, 2016.

SUMMARY: 3D body scanning technology is nowadays widely available and used in various research on body morphology and anatomical structure. The present study investigated the reliability and validity of NX-16 (TC2) 3D body scanner with a method of classical anthropometry. Research was carried out on 31 participants (17 males and 14 females) with their age $22.1 \pm 4.63$ years, their height $177.17 \pm 8.96 \mathrm{~cm}$ and their weight $72.36 \pm 13.6 \mathrm{~kg}$. Left (L) - Right (R) upper arm girth, L - R elbow girth, L - R forearm girth, L - R wrist girth, L - R thigh girth, L - R knee girth and L - R calf girth were measured with a flexible and inextensible tape with a $1 \mathrm{~mm}$ accuracy, and with the 3D measurement technique. The results show a good agreement between both methods used with paired variables showing significant and very strong correlations with more than $90 \%$ of shared variance; Bland-Altman plot also shows a high agreement; paired sample T-test significance was observed in most cases; no significant differences were observed in measurements of right wrist girth, left thigh girth, left knee girth and in left calf girth. NX-16 3D body scanner thus represents a valid and highly accurate tool for assessing human body dimensions. However, the 3D body scanning and classical anthropometry method cannot be regarded as interchangeable due to the different initial positions of the body in the implementation of measurement protocols. For the direct comparison of 3D scanning and the classical anthropometry method in the future, the same body starting position should be used as is held during the $3 \mathrm{D}$ scanning process.

KEY WORDS: Morphology; Anthropometry; Circumferences.

\section{INTRODUCTION}

Anthropometric characteristics are important biological variables related to sport performance (SánchezPuccini et al., 2014; Ferreira João \& Fernandes Filho, 2015). The ability to obtain and use precise anthropometric information regarding the physiological status of athletes is one of the fundamental issues in contemporary sport (Mikulic, 2008). Anthropometry is considered to be one of the most classical methods for assessing the dimensions of body segments (Heyward \& Wagner, 2004). The obtained results may vary due to the level of measurer's skills, as well as time-consuming procedures and measurement protocols, thus making the traditional anthropometry impractical in certain circumstances (Zancanaro et al., 2015). The recent digital shape-analysis tools are not limited to the traditional one-dimensional measurements, but instead they enable measuring the complex geometrical features (i.e. curvatures and partial volumes) (Bragança et al., 2014). With advancement of technology and application of 3D body scanners, methods for obtaining anthropometric body data have become more practical, contactless, fast and, above all, accurate
(Simmons \& Istook, 2003; Zhang et al., 2014; Ryder \& Ball, 2012; Bragança et al.). The 3D scanning methods were frequently used in textile industry (Apeagyei, 2010; Troynikov \& Ashayeri, 2011), in sports (Schranz et al., 2010; Simenko \& Vodicar, 2015), in healthcare (Treleaven \& Wells, 2007; Sims et al., 2012) and national surveys of general population (Wells et al., 2015). Some advantages of the 3D scanning represent a rapid raw data collection, a wide variety of digital shape outputs that can extend to $2 \mathrm{D}$ or 3D format, an electronic archiving of scans, which could be utilized in future analysis with improved software, a construction and comparison of composite shape models etc. (Wells et al., 2015).

Nowadays, there are many 3D scanning manufacturers and models using different scanning technologies. Zhang et al. compared the low cost 3D body scanner (KX16; TC2, Cary, NC) and tape measurements of mid-arm, fore-arm, thigh and ankle circumferences, and the results showed high correlations $(r-=0.89,0.69,0.90$, and 0.93 , respectively; all $\mathrm{p}<0.01)$ with non-significant

\footnotetext{
* Lecturer, Institute of Sport, Faculty of Sport, University of Ljubljana, Gortanova 22, 1000 Ljubljana, Slovenia.

${ }^{* *}$ Full professor, Department of Gymnastics, Faculty of Sport, University of Ljubljana, Gortanova 22, 1000 Ljubljana, Slovenia.
} 
Bland-Altman plots. Gropper et al. (2012) used NX-16 3D scanner in a 4-year study of 131 college students with regards to changes of weight, composition and shape. Wells et al. (2015) used classical anthropometry and the NX-16 3D body scanner (TC2, Cary, NC) to measure and obtain data of chest and waist girth, width and depth, and knee and calf girths on a sample of 1484 children aged 5-11 years. They reported that all manual measurements were successful, while 3D scans were successful in only 70.7 $\%$ of children. Unsuccessful scans were reported primarily due to body movement or the inability of the software to extract shape outputs. Measurements with the NX-16 3D body scanner tended to be greater than those obtained manually $(\mathrm{p}<0.05)$, however ranking consistency was reported to be high ( $\mathrm{r} 2>0.90$ for most outcomes). However, we are to conclude that they used the NX-16 scanner in an uncontrolled environment, because of transportation, placement and use of the NX-16 3D body scanner in a customized trailer, which was driven to a series of schools in order to collect data during school hours. Wells et al. (2015) did not report on the data about calibration, which are very important, since according to the manufacturer manual $(\mathrm{Ng}, 2009)$, every new installation of the scanner requires a full calibration. This is especially important, since there is no guarantee that a trailer was parked on a perfectly even terrain and due to the fact that the NX-16 is not a mobile model. The 3D body scanner NX-16 (TC2, Cary, NC) uses white light technology to obtain body measurements with a point accuracy of $<1 \mathrm{~mm}$ and a circumference accuracy of $<3 \mathrm{~mm}$, which represents a higher level of accuracy than would be achieved with traditional circumference measurements (Gropper et al., 2009).

With this in mind, we reviewed the available literature and concluded that there is a lack of studies, which would verify the reliability and validity of the NX-16 3D body scanner ([TC]2, Cary, North Carolina) in a controlled environment. Therefore, in this study we seek to verify the NX-16 3D body scanner's reliability and validity in comparison to the classical anthropometry method.

\section{MATERIAL AND METHOD}

Subjects. This study included 31 participants, of which 17 male and 14 female participants (both genders were chosen to make anthropometric variable variance bigger). Their mean age was $22.1 \pm 4.63$ years, their height $177.17 \pm 8.96 \mathrm{~cm}$ and their weight was $72.36 \pm 13.6 \mathrm{~kg}$. Written informed consent was obtained from all participants.
Anthropometric measurements: Body height was measured with anthropometer GPM (Switzerland). Left (L) - Right (R) upper arm girth, L - R elbow girth, L - R forearm girth, L - R wrist girth, L - R thigh girth, L - R knee girth and L - R calf girth were measured with a flexible and inextensible tape with a $1 \mathrm{~mm}$ accuracy, according to international biological program (IBP) (Lohman et al., 1988). Thus, IBP's basic rules and principles relating to the choice of parameters, standard conditions and measurement techniques were followed.

3D body scan measurements: 3D anthropometric measurement of body was performed by the 3D body scanner NX-16 ([TC]2, Cary, North Carolina). The NX-16 utilizes a non-invasive scanning method to produce a trueto-scale 3D body model in 8 seconds (Apeagyei). The scanner uses photogrammetry technology, which projects patterns of structured white light on to the body. The way in which the pattern is distorted by the shape of the body is then recorded by 32 cameras. From this, the body shape is digitally reconstructed from a raw photonic point cloud data which leads to a surface reconstruction of the body and allows for automatic landmark recognition as well as electronic tape measurements.

Experimental procedure. Subjects were measured by the same examiner with extensive experience in the physiological laboratory of the Faculty of Sport, University of Ljubljana in controlled environment conditions. Classic anthropometry was measured first and 3D body scan measurements were obtained afterwards.

Before measurements, we made full calibration of the NX-16 scanner. Full calibration was done using 1) the reference cylinder which was $150 \mathrm{~cm}$ in height and had diameter of $28 \mathrm{~cm}$, and 2) additional set of reference balls which included two 2 strings of calibration balls and a single calibration ball (diameter of all balls was $15 \mathrm{~cm}$ ). The scanner calibrated itself in a way that it measured a circumference on every $10 \mathrm{~mm}$ from top to bottom of the cylinder and calculated circumferences standard deviation that should not had exceeded prescribed limits of $0.9 \mathrm{~mm}$ $(\mathrm{Ng})$. Calibration with string of balls was successful and within the acceptable range of the circumferences standard deviation of $0.567 \mathrm{~mm}$.

Further, subjects were instructed to remove all jewelry and clothes. They entered the scanner barefooted and in form-fitting bright color underwear. They stood in a standardized position, with their feet located on landmarks on the scanner's floor (feet set straight, not inwards or outwards), grabbing the handles inside of the scanner with a natural standing posture (shoulders not 
elevated, elbows stretched, upright position of the back, chin slightly lifted). Subjects with long hair were instructed to tie their hair in a bun.

A 3D Body Measurement System Version 7.4.1 software was used to create the initial point cloud that was then processed into a 3D body model from which customized measurements could be extracted. A multi-scan option with 3 consecutive scans was used to obtain the data. Multi-scan options merged all 3 files of 3 consecutive scans and gave 1 merged file with means of all 3 consecutive scans. Scanning of 3 consecutive scans lasted $24 \mathrm{~s}$ and subjects were instructed to be still as much as possible.

Statistical Analysis. Analyses were conducted using SPSS for Windows (Version 21.0; SPSS, Inc., Chicago, USA). Data were presented according to descriptive statistics (Means \pm SD). Furthermore, we performed the following tests: Kolmogorov-Smirnov test, coefficient of variation $(\mathrm{CV})$, standard error of measurement (SEM), pairedsample T-test, Pearson correlation, coefficient of determination (R2), Cronbach's Alpha, Bland-Altman (Bland \& Altman, 1986) and average relative error. Relative error was calculated as absolute difference between 3D scanning method and classical anthropometry (hereinafter CA) result and divided with classical anthropometry result, and at the end average relative error was calculated. BlandAltman method of assessing agreement (Bland \& Altman) was calculated with the MedCalc software (Version 14.8.1; MedCalc $^{\circledR}$, Belgium). For calculating Bland-Altman figures we subtracted classical anthropometry values from the values obtained by the 3D body scanner (hereinafter 3D BS). All statistical significance for t-test, Pearson correlation and Cronbach's alpha was set to $\mathrm{p}<0.05$.

\section{RESULTS}

Kolmogorov-Smirnov test defined all variables as normal. Obtained parameters from both measuring methods were statistically compared and are presented in Table I. Differences were noticed depending on the measuring technique. In general, the two sets of results showed good agreement. Paired sample T-test significance was observed in most cases; no significant differences were observed in measurements of right wrist girth, left thigh girth, left knee girth and of left calf girth. Coefficients of variation were similar between the 3D scanner and hand anthropometry measurements. In general, standard error of measurements was small for both measurement techniques. Person correlations between pairs were significant and very strong with more than $90 \%$ of shared variance. Bland-Altman plot's (Figs. 1 and 2) showed high agreement between both methods of measurements.

\section{DISCUSSION}

The 3D body measurements showed high ranking consistency with manual anthropometry in adults. According to previous studies, 3D scanners provided with slightly larger, but systematical values (Wang et al., 2006; Wells et al., 2007; Wells et al., 2015), since reducing measurement errors to a minimum is highly important for both traditional and 3D anthropometry (Zancanaro et al., 2015).

In our study the slightly larger values from 14 variables measured on left and right body parts were equally divided between the 3D method and CA in exactly half of variables. 3D obtained values were greater in left and right upper arm girth, left and right wrist girth, right thigh girth, right knee girth and left calf girth. Upper arm girth measured by 3D BS was systematically greater. This is because in CA and according to the IBP protocol, the arms have to be in relaxed position beside the body and muscles forming the upper arm (biceps brachii and triceps) are thus relaxed. When conducting the 3D BS, the examinee needs to have his arms abducted (with active adduction muscles) to hold the scanner's handles in order to prevent unnecessary body movement and therefore the arm muscles and their tendons are slightly activated, resulting in greater upper arm muscles girths. Also the wrist girths were shown to be systematically greater when measured with 3D BS. The latter could also be explained with aforementioned arm position because in 3D BS one has to hold the handles and therefore the forearm muscles and tendons around the measuring position of the wrist girth are slightly activated resulting in higher girths when the 3D method is used. Slightly higher girths could also be explained when measuring calf girths. In CA and IBP protocol one is sitting on the bench and his/her leg is relaxed, not touching the floor, when the measurements are conducted. In 3D BS one is in standing position and calf muscles, which are also antigravity muscles, are activated resulting in higher calf girths. Thus, we conclude that the starting body position-parameters of 3D BS were slightly different when compared to CA with higher muscle tension, thus resulting in higher muscles girths, which was also reported by Wang et al. Wang et al. also noted that systematically higher values of 3D BS could be attributed to the accuracy of the CA method, which involved slight tension in manual measurements, as the tape is usually held over the skin. They reported that the disagreement between manual and 3D measurements decreased when a mannequin was measured instead of a human body, where the measurer could not use 
SIMENKO, J. \& CUK, I. Reliability and validity of NX-16 3D body scanner. Int. J. Morphol., 34(4):1506-1514, 2016.

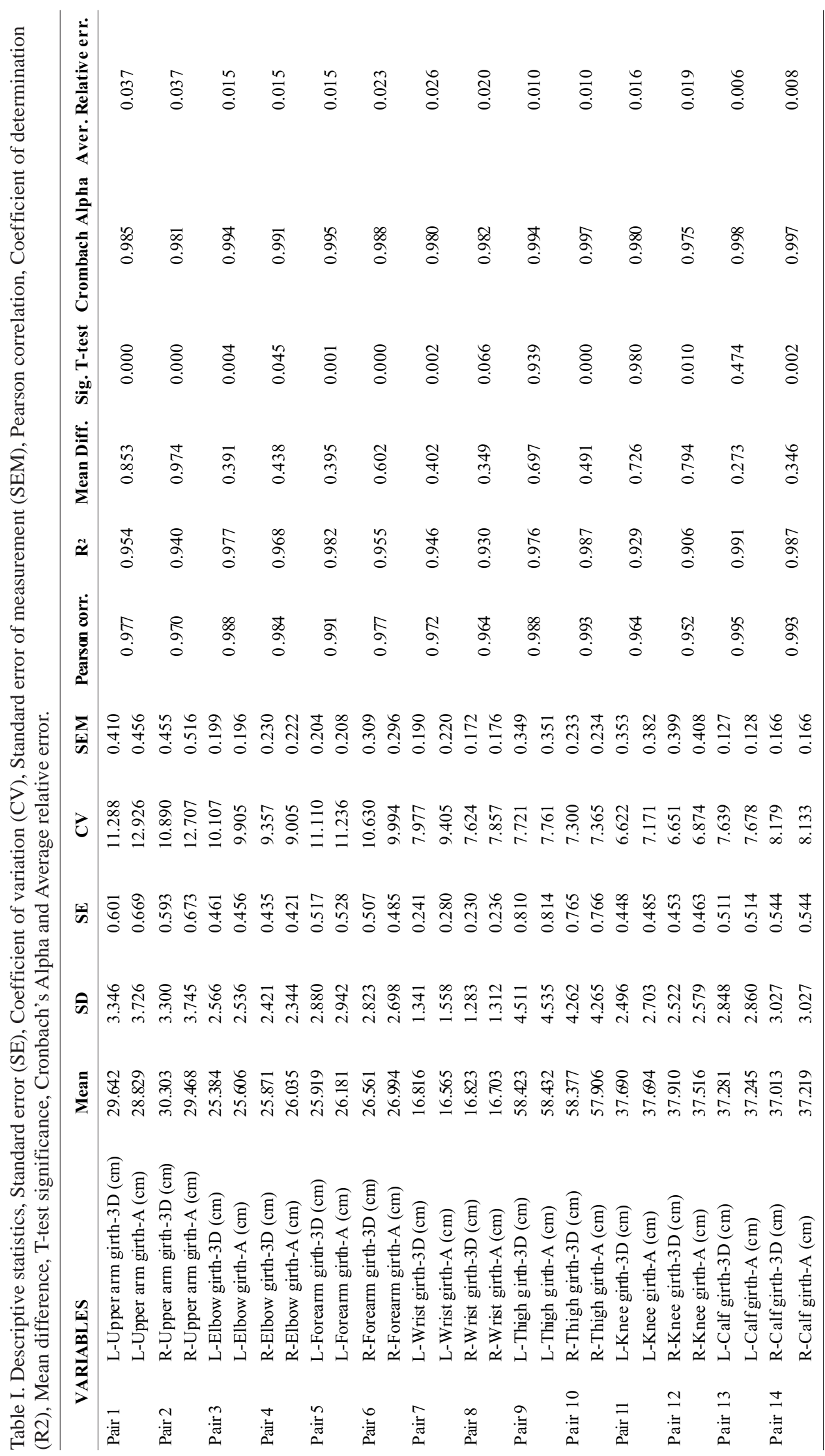

tape tension to change the dimension of the mannequin. This could also be used to explain significant differences between methods for the paired sample T-test, since 10 of 14 pairs were significantly different, but with non-significant BlandAltman plots. Again, this showed high agreement between both methods of measurements. The arm placement in the 3D BS could also be considered to be a contributing factor to the difference in higher upper arm girths.

According to the manufacturer's manual (Ng), arms should hold the handles and the elbows should be straight as possible, but there were no instructions on the upper arms position according to upper body torso. As we can see in Figure 4 upper arm cloud data could be grouped with upper torso cloud data (Fig. 4-B), thus resulting in higher cloud data acquisition and higher upper arm girths (Fig. 4-B1). This could be an issue when measuring obese population or athletes with big latissimus dorsi muscle. We recommend that the special attention is placed in instructions and demonstration prior to $3 \mathrm{D}$ scanning, emphasizing that the upper arm is not to touch with the upper torso as shown in Fig. 4-B2, which will provide with results with more accurate extraction of data as represented in Fig. 4B3. In order to increase the accuracy of thigh girth measurements, one should pay attention to very large 

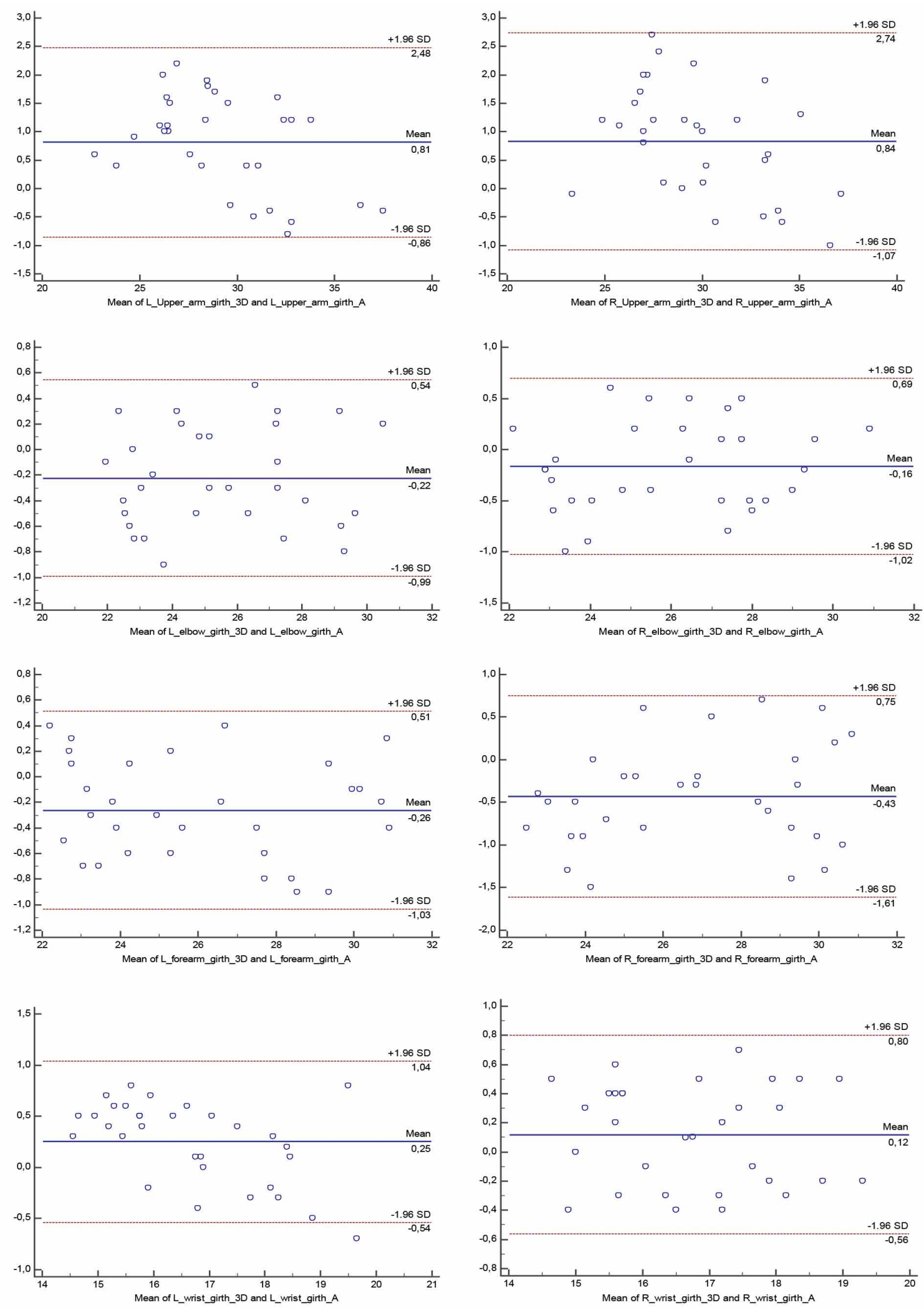

Fig. 1. Bland-Altman plots for the L-R upper arm girth, L-R elbow girth, L-R forearm girth and L-R wrist girth between the 3D body scanner NX-16 and classical anthropometry. 

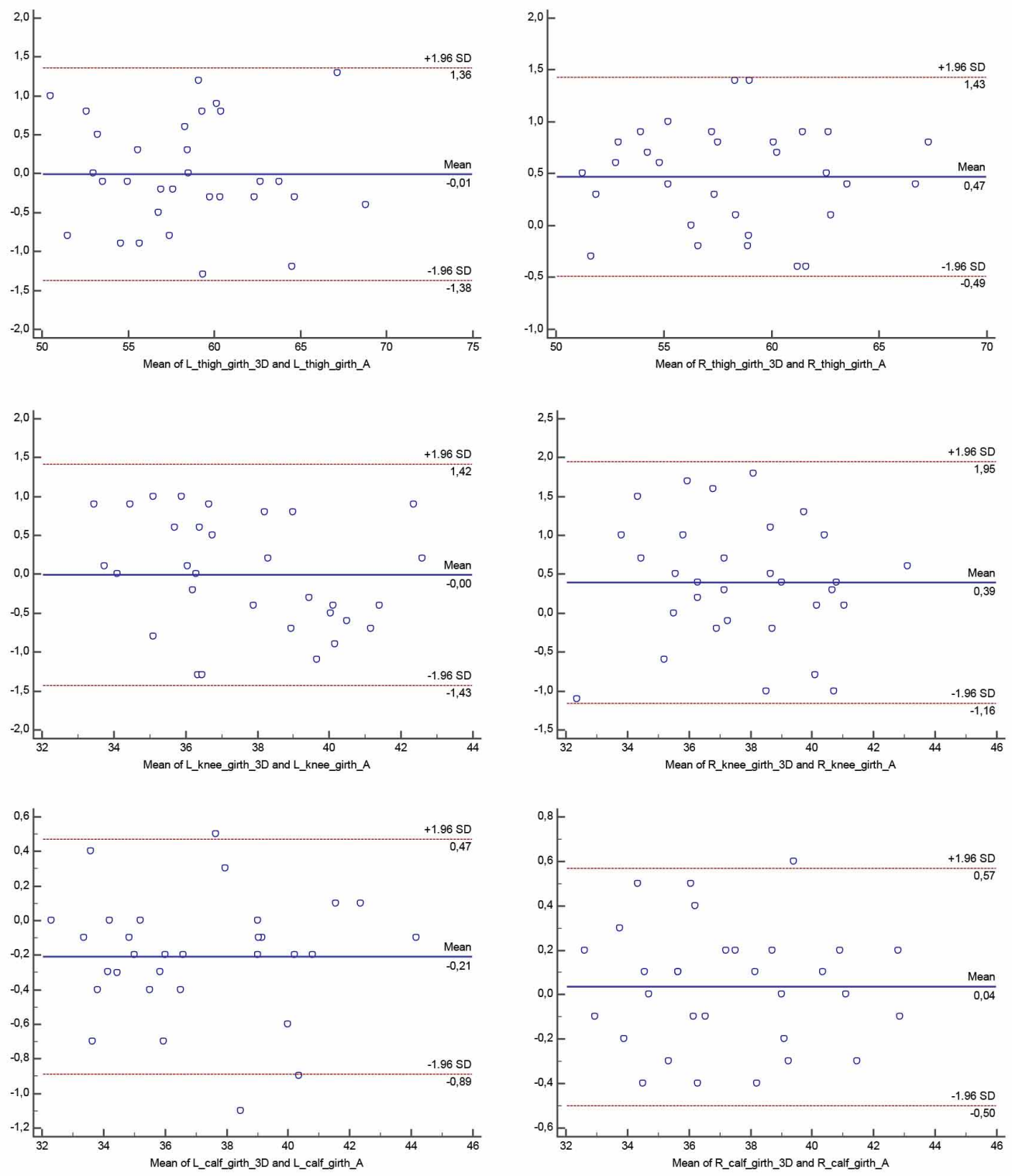

Fig. 2. Bland-Altman plots for the L-R thigh girth, L-R knee girth and L-R calf girth between the 3D body scanner NX-16 and classical anthropometry.

thigh muscles when measuring obese subjects or athletes. According to manufacturer instructions $(\mathrm{Ng})$, a subject is instructed to step on the two footprints on the carpet. However, when one measures obese subjects or subject with large thigh muscles, there is a possibility that the inner thigh scanned point cloud data will be merged as shown in Fig. 5$\mathrm{C}$. This will then result in inaccurate extraction of thigh girth data as shown in Fig. 5-C1. To avoid these errors, we recommend making a wider step on the outer side of the two footprints on the carpet. This wider position will assist in producing of a clearer cloud data image as shown in Fig. 5-C2 and more accurate extraction of thigh girth data as represented in Fig. 5-C3.
Furthermore, during the scanning process user could encounter some errors that could resolve in either unsuccessful scan or in successful one with errors in cloud data image of the scanned subject leading to wrong results. On the one hand, dark color tones of underwear and greater movements during the scan are considered to represent the errors leading towards unsuccessful scan clouds (Wells et al., 2015). On the other hand, there is a possibility of a successful extraction of the data with some possible errors in the shape of $3 \mathrm{D}$ point cloud data image. The latter could emerge if a scanned subject is wearing a non-form fitting underwear. These are considered to be software errors, since Software failure to extract body shape or body 

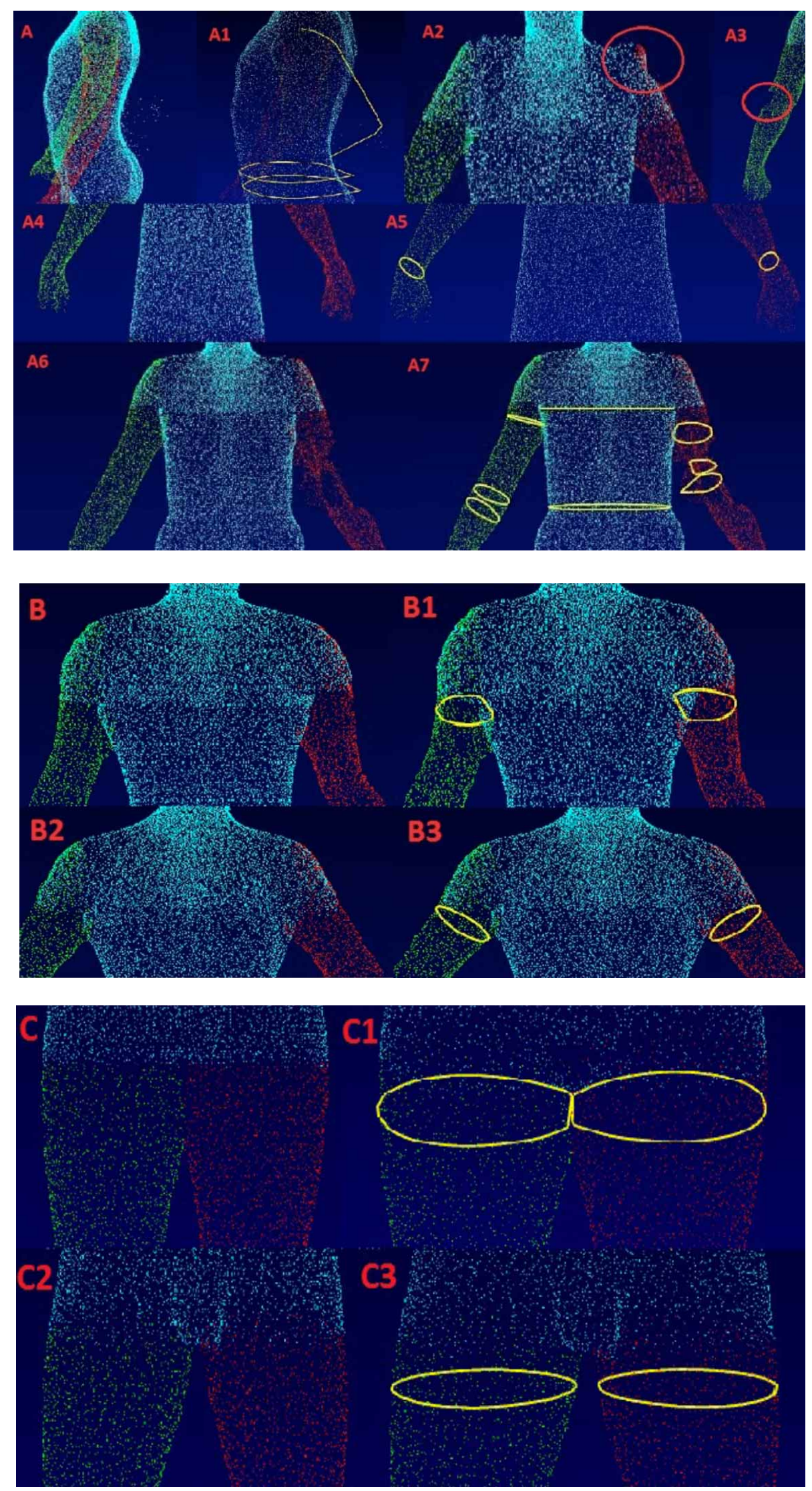

1512
Fig. 3. Possible software errors of cloud data with successful data extraction according to 3D software: A) Cloud data of 3D scanner with additional spatial dots; A1) Successful extraction of data despite additional spatial dots; A2) Cloud data error caused by movement during the scan; A3) Software error not producing the full cloud data body image on forearm; A4) Software error not producing the full cloud data body image on wrist; A5) Successful extraction of data despite Software error not producing the full cloud data body image on wrist; A6) Software error not producing the full cloud data body image on upper arm and forearm; A7) Successful extraction of data despite Software error not producing the full cloud data body image on upper arm and forearm.

Fig. 4. B) Usual placement of arms according to manufacturer instructions; B1) Possible error when extracting the data after usual placement of arms; B2) Our suggested placement of arms to reduce measurement error of upper arm girth; B3) Example of more accurate extraction of data after suggested arm placement.

Fig. 5. C) Usual placement of legs according to manufacturer instructions; C1) Possible error when extracting the data after usual placement of legs; C2) Our suggested wider placement of legs to reduce measurement error of thigh girth; C3) Example of more accurate extraction of data after suggested leg placement. 
measurements can be attributed to insufficient tailoring of the computer algorithms (Wells et al., 2015). Similar error could be also present when measuring small children, as the handles of the scanner are usually not suited for their height and the possibility of movement during the scan is therefore greater. These cloud data software problems could also occur randomly and could represent inability to locate leg girths or in additional movement of the arms during the scan (Wells et al., 2015).

In our study we encountered some additional errors in cloud data image, which are shown in Fig. 3. These need additional measurer's attention when conducting the scan, especially when extracting the data. Fig. 3-A represented a possibility of the scanner to detect some additional spatial dots in the space between the cameras and a scanned body, which could lead to wrong torso girths as shown in Fig. 3A1. In order to detect this error, an examiner needed to rotate the cloud data image from frontal plane to sagittal plane, because from a frontal plane this error was hard to detect. This error could be caused by some dust particles in the air thus reflecting back to the camera during scan. If the scanned person was moving in the shoulder joint, a possible cloud data error could occur as shown in Fig. 3-A2. Incomplete cloud data image could emerge in the area of forearm and elbow (Fig. 3-A3). This error could impact results of elbow and forearm girth if examiner would not be attentive to this error. Incomplete cloud data image could also emerge in the area of the wrist leading to the smaller wrist girths as represented in Fig. 3-A4 and Fig. 3-A5. Also blurred and incomplete cloud data may appear in arms (Fig. 3-A6 and Fig. 3-A7). All of above mentioned errors could be resolved with a repeated scan or by restarting the 3D body measurement software and repeating the scan. Since a single scan lasts only 8 seconds, it is not time consuming to repeat a scan and correct the occurred errors.

The advantage of 3D scanning is also in quicker operator training to conduct 3D anthropometry versus traditional anthropometry as Zancanaro et al. (2015) reported excellent intra-operator relative and absolute reliability in 3D measurement items independently of the operator's skills. However, in order to ensure a high precision of $3 \mathrm{D}$ scans, we recommend that a trained operator conducts the scan. Thus, a quick detection of possible errors on the cloud data image could be ensured, especially when a scanner does not report a failed scan and a visual inspection of the cloud data image is necessary. For the higher accuracy, it is necessary to pay attention on position of arms and legs during a scan.

In general, our results show high agreement of the 3D BS and CA method with very high correlations among them and with excellent internal consistency shown by Cronbach Alpha. Therefore, we can conclude that the 3D BS technique represents a valid and reliable tool for the measurement of morphological data. It provides with high accuracy when correctly used and it helps in acquiring data that could be used in talent identification, which usually could not be assessed by using conventional anthropometry (Schranz et al.; Ackland et al., 2012). Hence, methods of the 3D body scanning and classical anthropometry could not be regarded as interchangeable, since there are differences in initial body positions due to the implementation of measurement protocols (Wells et al., 2015). Therefore, in order to provide with a direct comparison of 3D BS and CA using Bland-Altman method, we suggest to pay special attention to body position, for which is imperative to be equal during both the CA measurement and 3D BS measurement in order to maximize the equality of both measurements conditions.

SIMENKO, J. \& CUK, I. Confiabilidad y validez del escaner corporal 3D NX-16. Int. J. Morphol., 34(4):1506-1514, 2016.

RESUMEN: La tecnología de escaner del cuerpo en 3D se encuentra ampliamente disponible y se utiliza en diversas investigaciones de la morfología del cuerpo y las estructuras anatómicas. El presente estudio investigó la fiabilidad y validez del escáner corporal NX-16 (TC2) 3D con un método de la antropometría clásica. La investigación se realizó en 31 participantes (17 hombres y 14 mujeres) con rango etario de $22,1 \pm$ 4,63 años, altura de $177,17 \pm 8,96 \mathrm{~cm}$ y peso de $72,36 \pm 13,6 \mathrm{~kg}$. Se midieron con una cinta métrica flexible e inextensible con precisión de $1 \mathrm{~mm}$, y con la técnica de medición 3D la circunferencia del brazo superior, I - D, circunferencia del codo, I - D, circunferencia de antebrazo, I - D, circunferencia de la muñeca, I - D, circunferencia del muslo, I - D y circunferencia de rodilla I - D. Los resultados mostraron una buena concordancia entre los dos métodos utilizados con variables que presentan correlaciones importantes y significativas, con más del $90 \%$ de la varianza compartida; Bland-Altman plot mostró importantes correlaciones. No se observaron diferencias significativas en las mediciones de la circunferencia de la muñeca derecha, la circunferencia del muslo izquierdo, la circunferencia de la rodilla izquierda y la circunferencia de la pierna izquierda. NX-16 escáner de cuerpo en $3 \mathrm{D}$ representa un número válido y altamente preciso para evaluar las dimensiones del cuerpo humano. El escaneo en 3D del cuerpo y el método de la antropometría clásica no pueden ser considerados como intercambiables debido a las diferentes posiciones iniciales del cuerpo en la aplicación de los protocolos de medición. Para la comparación directa de escaneado 3D y el método de la antropometría clásica en el futuro, se deberá utilizar la misma posición del cuerpo, que se lleva a cabo al inicio del proceso de digitalización en 3D.

PALABRAS CLAVE: Morfología; Antropometría; Circunferencias. 


\section{REFERENCES}

Ackland, T. R.; Lohman, T. G.; Sundgot-Borgen, J.; Maughan, R. J.; Meyer, N. L.; Stewart, A. D. \& Müller, W. Current status of body composition assessment in sport: review and position statement on behalf of the ad hoc research working group on body composition health and performance, under the auspices of the I.O.C. Medical Commission. Sports Med., 42(3):227-49, 2012.

Apeagyei, P. R. Application of 3D body scanning technology to human measurement for clothing Fit. Int. J. Digit. Content Technol. Appl., 4(7):58-68, 2010.

Bragança, S.; Carvalho, M.; Xu, B.; Arezes, P. \& Ashdown, S. A Validation Study of a Kinect Based Body Imaging (KBI) Device System Based on ISO 20685:2010. Conference. Lugano, 5th International Conference on 3D Body Scanning Technologies, 2014.

Bland, J. M. \& Altman, D. G. Statistical methods for assessing agreement between two methods of clinical measurement. Lancet, 1(8476):30710, 1986.

Gropper, S. S.; Simmons, K. P.; Gaines, A.; Drawdy, K.; Saunders, D.; Ulrich, P. \& Connell, L. J. The freshman 15-a closer look. J. Am. Coll. Health, 58(3):223-31, 2009.

Gropper, S. S.; Simmons, K. P.; Connell, L. J. \& Ulrich, P. V. Changes in body weight, composition, and shape: a 4-year study of college students. Appl. Physiol. Nutr. Metab., 37(6):1118-23, 2012.

Heyward, V. H. \& Wagner, D. R. Applied Body Composition Assessment. 2nd ed. Champaign, Human Kinetics, 2004.

Ferreira João, A. \& Fernandes Filho, J. Somatotype and body composition of elite brazilian gymnasts. Sci. Gymnast. J., 7(2):45-54, 2015.

Lohman, T. G.; Roche, A. F. \& Martorell, R. Anthropometric standardization reference manual. Champaign, Human Kinetics Books, 1988.

Mikulic, P. Anthropometric and physiological profiles of rowers of varying ages and ranks. Kinesiology, 40(1):80-8, 2008.

Ng, J. NX-16-Training \& Installation. Singapore, Tridigen Private Limited, 2009.

Ryder, J. R. \& Ball, S. D. Three-dimensional body scanning as a novel technique for body composition assessment: A preliminary investigation. J. Exerc. Physiol. Online, 15(1):1-14, 2012.

Sánchez-Puccini, M. B.; Argothy-Bucheli, R. E.; Meneses-Echávez, J. F.; López-Albán, C. A. \& Ramírez-Vélez, R. Anthropometric and physical fitness characterization of male elite karate athletes. Int. J. Morphol, 32(3):1026-31, 2014.

Schranz, N.; Tomkinson, G.; Olds, T. \& Daniell, N. Three-dimensional anthropometric analysis: differences between elite Australian rowers and the general population. J. Sports Sci., 28(5):459-69, 2010.

Sims, R.; Marshall, R.; Gyi, D. E.; Summerskill, S. \& Case, K. Collection of anthropometry from older and physically impaired persons: traditional methods versus TC2 3-D body scanner. Int. J. Ind. Ergon., 42(1):65-72, 2012.
Simmons, K. P. \& Istook, C. L. Body measurement techniques: A comparison of three-dimensional body scanning and physical anthropometric methods for apparel application. J. Fash. Mark. Manag., 7(3):306-32, 2003.

Simenko, J. \& Vodicar, J. Evaluation of Body Symmetries in Judo. In: Doupona Topic, M. \& Kajtna, T. (Eds.). Youth Sport: Proceedings book / 7th Conference for Youth Sport, Ljubljana, 12-13 December 2014. Ljubljana, Fakulteta za Sport, 2015. pp.38.

Treleaven, P. \& Wells, J. 3D body scanning and healthcare applications. Computer, 40(7):28-34, 2007.

Troynikov, O. \& Ashayeri, E. 3D Body Scanning Method for Close-Fitting Garments in Sport and Medical Applications. Melbourne, Ergonomics Australia - HFESA 2011 Conference Edition, 2011. pp.11-6.

Wang, J.; Gallagher, D.; Thornton, J. C.; Yu, W.; Horlick, M. \& Pi-Sunyer, F. X. Validation of a 3-dimensional photonic scanner for the measurement of body volumes, dimensions, and percentage body fat. Am. J. Clin. Nutr., 83(4):809-16, 2006.

Wells, J. C.; Treleaven, P. \& Cole, T. J. BMI compared with 3-dimensional body shape: the UK National Sizing Survey. Am. J. Clin. Nutr, 85(2):419-25, 2007.

Wells, J. C.; Stocks, J.; Bonner, R.; Raywood, E.; Legg, S.; Lee, S.; Treleaven, P. \& Lum, S. Acceptability, precision and accuracy of 3D photonic scanning for measurement of body shape in a multiethnic sample of children aged 5-11 years: The SLIC Study. PloS One, 10(4): $\mathrm{e} 0124193,2015$

Zancanaro, C.; Milanese, C.; Lovato, C.; Sandri, M. \& Giachetti, A. Reliability of three-dimensional photonic scanner anthropometry performed by skilled and naïve operators. Int. J. Ergon., 5(1):1-11, 2015.

Zhang, K.; Zheng, J.; Gao, C.; Thomas, D.; Li, X. \& Heymsfield, S. Rapid-accurate anthropometric body shape assessment with lowcost novel 3D imaging system. F. A. S. E. B. J., 28(Suppl. 1):391.2, 2014.

\section{Correspondence to: \\ Jozef Simenko \\ Institute of Sport \\ Faculty of Sport, University of Ljubljana \\ Gortanova 22, 1000 Ljubljana \\ SLOVENIA}

Email: jozef.simenko@fsp.uni-lj.si

Received: 21-04-2016

Accepted: 20-09-2016 